\title{
Study on the Experimental Teaching Mode for Computer MOOC
}

\author{
Xie-Qiu ZHAO ${ }^{1}$, Yan-Ming CHENG ${ }^{2 *}$ Xi-Jiao LI ${ }^{1}$ \\ ${ }^{1}$ College of Computer Science \& Technology Engineering, Beihua University, China \\ ${ }^{2}$ College of Electrical \& Information Engineering, Beihua University, China \\ ${ }^{*}$ Corresponding author
}

Keywords: MOOC; online experiment teaching; experiment teaching mode

\begin{abstract}
.
With the rapid development of computer technology, Students majoring in computer science have a difficulty in shifting the theoretical knowledge to practical works which impacts the employment. How to improve the manipulative ability of students results in higher demand on the computer experiment. In the shortest period, more students are allowed to improve their practical ability through online experiment teaching, the massive open online course (MOOC) appears that provides a better solution for us in the experiment teaching mode.
\end{abstract}

\section{Introduction}

With increasing development of the modern computer technology and the demand of talents, higher requirements are put forward for college students of computer major in computer science experiment. The traditional experimental teaching has been unable to meet the needs of school experiment, consequently, the massive open online course (MOOC) was proposed, which is a revolution in higher education is shown in Fig.1. Computer software and hardware experiments can be conducted by MOOC, at the same time, more experimenters will benefit from the online experiment system.

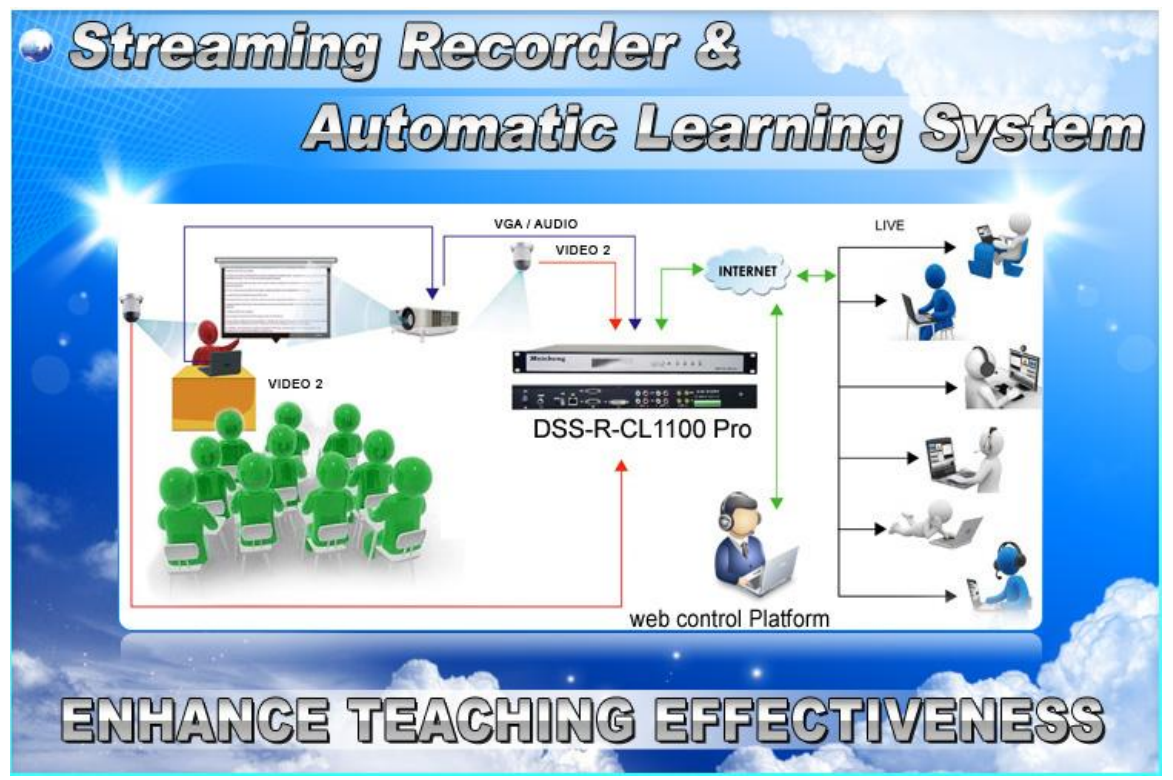

Fig.1 Online teaching scenario

\section{The Importance and Characteristics of Computer Experiment Teaching}

Computer science and technology, software engineering, network engineering same as all other engineering disciplines, requires students not only to master solid theoretical professional knowledge, but also to experience more practices. Experimental teaching is a indispensable part in the whole teaching process, whether it is a theory of computer software or hardware, which must help students 
understand and master the practical operation through effective experimental teaching, which can train students' team spirit and scientific innovation consciousness.

At present, the traditional experimental teaching has the following characteristics:

1) The need for class hours guarantee

The adequate teaching theory as the basis, experimental teaching requires enough class time guarantee, only students well experience practice, they can better understand the theory. Students often meet some questions when they do experience in the practice teaching, teachers must directly guide in person, however, the practice teaching for the traditional computer practice must be completed under experimental conditions, especially the need of hardware equipment to complete the experiment, and students must complete in the laboratory. It is necessary to ensure that there is adequate hardware and adequate time to ensure that the experiment teaching.

2) The need for investment in a large number of teachers

After the completion of theoretical teaching, the experimental teaching needs teachers pay more time and efforts. Before experiment teaching, teachers need do repeated experimental verification of the theory and, which will takes a lot of time in the hardware debugging to ensure the smooth conduct of the experiment. During the experiment process, students might encounter various problems; therefore, teachers need to solve corresponding dealing ways according to these problems, which requires teachers put more efforts. The university is also equipped with experimental teachers assist experimental guidance, which increases the investment in teachers.

3) A large number of equipment investments

The computer experimental teaching practice requires a lot of equipment support, such as computer software need to install a lot of software and hardware of experimental instruments and equipment many need to cooperate, let the students hands-on experiments to verify the relevant content, a more intuitive understanding of the theory, the purchase of equipment requires a lot of money, but also requires a lot of maintenance manpower and materials.

These traditional teaching require a major investment in human and material resources, the majority of colleges and universities strengthen as far as possible the experimental teaching links in all aspects to ensure the effective completion of experimental teaching. Therefore, online experiment is put forward.

\section{Online Experiments}

With the requirements and development of computer experimental, higher requirements on the experimental teaching is required to improve college students' practical ability, which results in the concept of MOOC. the assessment for students is implemented by the combination with the online operation and normal test, online forums and the offline final exam in the class. while the theory teaching, with live video and handouts, the students have the feeling personally on the scene in the study; teachers as well as can communicate with students online through the forum form and student course answering, which can more effectively complete the task of theory teaching, and the complete multi evaluation including self-assessment, peer assessment, students assessment.

\section{The Solution of Computer Experiment in Online Education}

For the solution of computer experiments, many colleges and universities conducted a series of exploration and attempt on online case including software and hardware experiments.

1) The software experiment is implemented by online or remote login server. In particular, the experimental teaching of computer software is mainly on the verification of experiments, comprehensive experiments, as well as the design of the program, the verification and evaluation of which can be automatically completed g via online evaluation system. Learners submit code to the online evaluation system to complete the examination and evaluation of the experiment, and evaluation system can complete duplicate checking for the design to ensure the fairness of each 
learner acknowledging experiment and experiment evaluation, which will reduce the amount of experimental work. Online experiment has been developed and can be applied to large-scale online courses to a certain extent.

2) Hardware experiment is a difficult, due to the hardware requires a lot of hardware equipment, in the course of the experiment, there are many complicated and difficult to control, which hinders the development of online hardware curriculum, therefore, many universities have put forward the solution of large-scale online courses, such as local experiment, simulation experiment, remote experiment. The local experiment is based on the traditional experimental methods, the first is to complete the theory learning, and then build an experimental platform to prepare the hardware equipment, and to verify the correctness of the experiment with regards to the online experiment guide, which can only meet the general experiment. The simulation experiment is achieved through the software simulating hardware environment shown in Fig.2.

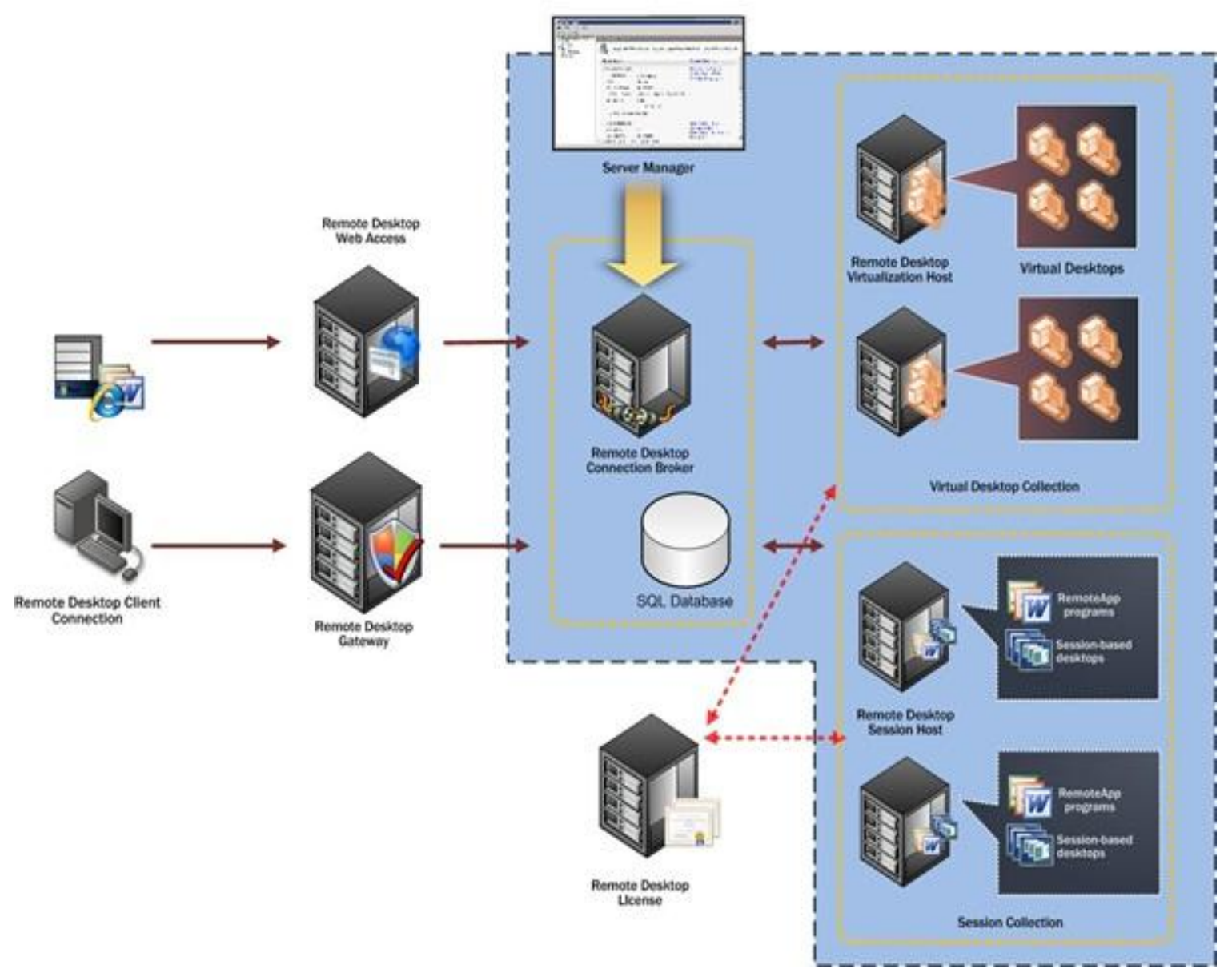

Fig.2 Structure of the computer experiment teaching

MOOC can achieve free access to resources, learner can also be free to use large open online courses under consideration of without school roll, and also there is no limit on the number of students, which can be implemented as long as students are the online. On basis of combining with the local hardware model, the experimental results can be obtained by network software controlling local hardware devices, thus, the experimenter complete online configuration of local hardware and check online results, which can achieve same effect as examination results in local experiment. In the online course experiments in a large scale, experiment can be dispersedly and randomly conducted, therefore, quantity demand of experiment equipment is not much, and the results will be stored in the online database, which can be check any time, at the same time the attempt at remote video teaching is being carried out. By comparing the three models, that is committed to solve the online experiment requirements of large-scale experiments, different characteristics, the requirements of different courses, but the remote experiment is most close to the experimental results of traditional 
experimental scheme, which is the future development direction, which can greatly reduce laboratory demand, workload of the teacher and the teacher, students can do the online experiment at any time under condition of arranging the time reasonably, and then the quality of experimental teaching success rate is improved, which will make the students understand and master the profession through the experiment, finally find the interest from experiment, improve the operational capacity.

\section{Conclusions}

By comparing the traditional experimental environment with online experiment, the realization of the MOOC mode is imperative. Especially in the rational use of resources, innovation and experiment, the use of big data era, cloud server continually improving, online simulation flat, the online experiment and the remote video teaching can be used in large-scale to motivate learning desire to acquire knowledge. It Realizes transform passive learning into active learning desire and completing the task of experimental teaching in free learning and happy learning.

\section{Acknowledgements}

This work was supported by the Scientific and Technological Planning Project of Beihua University, China (No. YB201620).

\section{References}

[1] Li Xiaoming, Peking University, MOOC research and practice summary , industry and information technology education, 2014 (11) 1-2.

[2] Lv Hong et al., Research and application of MOOC Based Computational Thinking oriented college computer experiment teaching reform, automation technology and application 2016 (12); 37-42.

[3] Li Ping, Computer experiment teaching reform and exploration based on cloud platform network and Information Engineering, 2016 (9) 93-94.

[4] Xu Xiaofei, Seize the opportunity of MOOC to promote the teaching reform of computer and software engineering, college education in China, 2014 (1); 29-33.

[5] Chi Yaqing, Song Ruiqiang, Exploring the impact of MOOC on the teaching of computer courses , Computer Engineering and science, 2014,63 (4); 164-168

[6] Li Shanshan, Ken Chan, Discussion on computer experimental teaching in MOOC Era, computer education 2014 (13) 103-106. 